\title{
Utilização de métodos invasivo e não invasivo na predição das performances aeróbia e anaeróbia em nadadores de nível nacional
}

\author{
Marcelo Papoti ${ }^{1,2}$ \\ Alessandro M. Zagatto ${ }^{2,3}$ \\ Olga C. Mendes ${ }^{1}$ \\ Claudio A. Gobatto ${ }^{2}$
}

\author{
${ }^{1}$ Faculdades Integradas de Bauru, SP, Brasil \\ ${ }^{2}$ Laboratório de Biodinâmica, UNESP Rio Claro,SP, Brasil \\ ${ }^{3}$ Universidade Federal do Mato Grosso do Sul, MS, Brasil
}

\section{RESUMO}

O objetivo do presente estudo foi comparar a velocidade crítica (VC) com o limiar anaeróbio (LAN) e verificar as relações dessas variáveis e da capacidade de nado anaeróbio (CTA) com o desempenho de nadadores nas distâncias de $15 \mathrm{~m}, 25 \mathrm{~m}, 50 \mathrm{~m}$, $100 \mathrm{~m}, 200 \mathrm{~m}$ e $400 \mathrm{~m}$ nado crawl. Participaram voluntariamente do estudo 8 nadadores de nível nacional, que realizaram 3 esforços progressivos (85\%, 90\% e 100\%) de $400 \mathrm{~m}$ nado crawl para determinação do LAN, bem como esforços máximos nas distâncias de $15 \mathrm{~m}, 25 \mathrm{~m}, 50 \mathrm{~m}, 100 \mathrm{~m}, 200 \mathrm{~m}$ e $400 \mathrm{~m}$ em estilo crawl. Os tempos obtidos nas distâncias de $200 \mathrm{~m}$ e $400 \mathrm{~m}$ foram submetidos ao procedimento de regressão linear para determinação da VC (coeficiente angular) e CTA (coeficiente linear). A VC $(1,38 \pm 0,07)$ não foi significativamente diferente $(\mathrm{p}<0,05)$ do LAN $(1,37 \pm 0,05)$ e apresentaram correlações significativas com a performance de $400 \mathrm{~m}$ nado crawl $(\mathrm{p}<0,05)$, enquanto que a CTA não se correlacionou significativamente com nenhuma das performances de nado. Desse modo pode-se concluir que a VC é um parâmetro confiável na avaliação da capacidade aeróbia e na predição da performance de $400 \mathrm{~m}$ nado crawl. No entanto, a CTA obtida pelo intercepto-y não foi um bom preditor da performance dos nadadores nas distâncias entre $15 \mathrm{~m}$ a $400 \mathrm{~m}$.

Palavras-chave: natação, capacidade anaeróbia, velocidade crítica, performance.

\begin{abstract}
Use of invasive and non-invasive protocol tests on aerobic and anaerobic performances prediction in Brazilian swimmers
\end{abstract}

The purpose of this study was to compare the critical velocity (VC) with the anaerobic threshold (LAN) and verify the relationship among $V C$ and LAN with anaerobic swimming capacity (CTA) with the swimmer's performance in the $15 \mathrm{~m}, 25 \mathrm{~m}, 50 \mathrm{~m}, 100 \mathrm{~m}, 200 \mathrm{~m}, 400 \mathrm{~m}$ distances in crawl swimming. Eight national level swimmers were volunteers in the present study, and performed 3 progressive efforts of $400 \mathrm{~m}$ crawl swimming $(85 \%, 90 \%$ and $100 \%)$ for LAN determination. They also performed the maximum effort in the $15 \mathrm{~m}, 25 \mathrm{~m}, 50 \mathrm{~m}$, $100 \mathrm{~m}, 200 \mathrm{~m}$ and $400 \mathrm{~m}$ distances in crawl style. The scores resulted from the $200 \mathrm{~m}$ and $400 \mathrm{~m}$ distances were submitted to the linear regression procedure for $V C$ determination (angular coefficient) and CTA (linear coefficient). The VC $(1.38 \pm 0.07)$ was not significantly different from the LAN $(1.37 \pm 0.05)$ and presented significant correlations with the $400 \mathrm{~m}$ crawl swimming performance $(p<0.05)$, while the CTA did not correlate significantly with any of these swimming performances. In this way, it is possible to conclude that the VC is a trustful reliable indicator for the evaluation of the aerobic capacity in swimming. However, the CTA resulted from y-intercept was not a good predictor of the swimmer's performance in the distances from $15 \mathrm{~m}$ to $400 \mathrm{~m}$ crawl swimming.

Key Words: swimming, anaerobic capacity, critical velocity, performance. 


\section{INTRODUÇÃO}

A determinação das concentrações sanguíneas de lactato é uma ferramenta amplamente utilizada por técnicos e pesquisadores para quantificar as cargas de treinamento $(28,34)$, monitorar a evolução dos nadadores (36) e prescrever as intensidades ideais do treinamento aeróbio com base na determinação do limiar anaeróbio (22). Essa determinação, resumidamente, consiste em submeter o nadador a esforços progressivos em distância previamente definida e intervalos relativamente curtos entre os esforços, com coletas de amostras sanguíneas após cada nado. Utilizando a relação entre a concentração de lactato ([La-]) versus a velocidade média de nado $(\mathrm{Vm})$, é possível determinar uma velocidade muito próxima à ideal para o treinamento aeróbio $(11,21)$. Dentre as técnicas amplamente utilizadas para determinação do limiar anaeróbio, se destacam a convencional, obtida pelo aumento abrupto do lactato, o aumento de $1 \mathrm{mM}$ na concentração de lactato acima da linha de base em testes de cargas contínuas, ou pela intensidade de exercício correspondente à concentração fixa de $4 \mathrm{mM}$ de lactato, além de outros. Recentemente, Pereira et al. (35) validaram dois protocolos para a determinação do limiar anaeróbio (LAN) em natação utilizando a concentração fixa de 3,5 mM de lactato, com a realização de 3 esforços progressivos nas distâncias de $200 \mathrm{~m}$ e $400 \mathrm{~m}$, intervalo de 3 minutos entre os esforços e coleta de amostras sanguíneas 1 minuto após cada nado. Esses protocolos foram comparados com a velocidade de máxima fase estável de lactato (MFEL). Os autores nesse estudo, além de não verificarem diferenças significativas entre os LANs determinados com a utilização de ambos os esforços (200m e $400 \mathrm{~m}$ ) e a MFEL, também observaram elevadas correlações $(p<0,05)$, evidenciando a validade desses protocolos na determinação da capacidade aeróbia de nadadores.

Embora todos os critérios utilizados, geralmente, apresentem elevadas correlações $(r>0,92)$ com a performance de endurance, os valores de LAN, dependendo do protocolo utilizado, podem diferenciar-se significativamente em até $15 \%$ (44), o que pode subestimar ou superestimar as intensidades do treinamento. No entanto, o lactato sangüíneo é uma ferramenta sensível a pequenas adaptações no treinamento de nadadores (36).
Os métodos para mensurar variáveis anaeróbias como força e potência não são tão bem desenvolvidos como os que avaliam as qualidades aeróbias, embora sejam aspectos importantes para a evolução do nadador (40). Maglischo (24) sugeriu, como forma de avaliar a capacidade anaeróbia, a determinação da concentração de lactato sanguíneo após esforços máximos, sendo que baixos valores de lactato, juntamente com desempenhos insatisfatórios, poderiam indicar a deterioração desta capacidade. No entanto, a diminuição da concentração sanguínea de lactato após esforços máximos pode ser devida a um estado de supertreinamento $(6,16,20,42)$. Também são freqüentes metodologias que avaliam a força dos nadadores, fora da água, utilizando o banco de nado "swim bench" (38) e, na água, em situação de nado atado $(3,13,17,23,25,29,32,33)$. Essa última, além de apresentar especificidade superior ao "swim bench", é um ergômetro reprodutível $(13,25,37)$, sensível aos efeitos do treinamento $(32,46)$ e altamente correlacionado com a velocidade de nado nas distâncias entre $25 \mathrm{~m}$ a $400 \mathrm{~m}$ nado $\operatorname{crawl}(25,33)$. Infelizmente, nem todas as equipes dispõem do suporte financeiro necessário para a aquisição de equipamentos específicos na mensuração da força e potência, ou para a realização constante de avaliações utilizando a lactacidemia. Por esses motivos, é crescente o número de estudos empenhados em desenvolver metodologias confiáveis e de baixo custo, a fim de proporcionar aos treinadores uma ferramenta segura para avaliação das capacidades físicas dos nadadores, assim como a prescrição e monitoramento das intensidades do treinamento. O teste de 30 minutos é um método clássico que fornece de maneira não invasiva a velocidade de nado correspondente ao limiar anaeróbio. Nesse teste, a velocidade média de nado obtida durante um esforço máximo de 30 minutos não foi significativamente diferente e ainda apresentou elevadas correlações com a velocidade de nado correspondente ao limiar anaeróbio determinado com a utilização da lactacidemia $(5,30)$. No entanto, o método é limitado por desconsiderar o componente anaeróbio envolvido, podendo não refletir sensivelmente as adaptações aeróbias dos nadadores. A utilização da potência crítica, proposta por Monod e Scherrer (26) e validada por Moritani et al. (27) - 
teoricamente representa a máxima carga de trabalho que pode ser mantida por um longo período de tempo sem fadiga - tem sido objeto de vários estudos, parecendo ser um método alternativo de avaliação bastante conveniente, não apenas por se tratar de um modelo não invasivo, mas também por fornecer, em um único teste, indicadores das capacidades aeróbia e anaeróbia dos atletas. Basicamente, esse procedimento consiste em aplicar exercícios com intensidade constante medindo-se o tempo até o indivíduo entrar em exaustão (tempo limite). Essa relação hiperbólica também pode ser utilizada para expressar a relação entre a velocidade e o tempo de esforço (31).

Wakayoshi et al. (48), utilizando o conceito "linearizado" de potência crítica, verificaram se a velocidade crítica (VC) pode ser utilizada para estimar a performance de nadadores competitivos. Nesse estudo, para determinação da VC e da capacidade de "nado" anaeróbio (CTA), os nadadores foram submetidos a seis esforços até a exaustão no "swimming flume". Os seis pontos obtidos da relação entre o tempo limite (Tlim) e a velocidade de nado (VN) foram submetidos ao procedimento de regressão linear, sendo que o coeficiente angular representou a VC e o coeficiente linear (intercepto-y) a CTA. Os autores observaram elevada correlação da VC com o limiar de lactato para concentração de $4 \mathrm{mM}(\mathrm{p}<0,01)$, com o limiar ventilatório $(\mathrm{p}<0,05)$ e com a velocidade máxima de $400 \mathrm{~m}(\mathrm{p}<0,01)$.

Em outro estudo, Wakayoshi et al. (49), utilizando o mesmo procedimento matemático descrito anteriormente, no entanto com distâncias pré-determinadas e assumindo que a relação entre a velocidade de nado e o tempo de esforço são lineares, constataram que a VC determinada em piscina convencional, apenas com a realização de dois esforços máximos em distâncias de $200 \mathrm{~m}$ e $400 \mathrm{~m}$, corresponde à máxima fase estável de lactato, sugerindo sua utilização na avaliação da performance física sem a necessidade de amostras sanguíneas, ou equipamentos sofisticados. Kokubun (19) verificou a relação da VC com o limiar anaeróbio e sua sensibilidade aos efeitos do treinamento em 48 nadadores. Foram determinados o limiar anaeróbio correspondente à concentração de lactado de $4 \mathrm{mM}$ e a velocidade crítica, obtida pela linearização da função hiperbólica, após o cálculo das velocidades médias dos nadadores, determinadas através da realização de três esforços máximos em estilo crawl (100, 200 e 400m). Esse pesquisador relatou ao final do estudo que o modelo matemático utilizado para determinar a VC é um bom parâmetro para avaliar a capacidade aeróbia dos nadadores. Como foi citado anteriormente, a CTA teoricamente corresponde à variável anaeróbia do modelo de VC, sendo representada pelo coeficiente linear (intercepto-y). Foi demonstrado que essa variável não é afetada pela hipóxia (Moritani et al., 1981), é sensível a 8 semanas de treinamento intervalado de alta intensidade (12) e a 6 semanas de treinamento resistido (1). Além disso, a CTA foi significativamente correlacionada com o teste de Wingate (9), produção anaeróbia de ATP muscular $(\mathrm{r}=0,70)$ em ciclistas bem treinados (8) e com o déficit de oxigênio (12), sugerindo que o intercepto-y pode ser um parâmetro válido, para representar a capacidade de trabalho anaeróbio $(9,14,15)$.

No entanto, os estudos encontrados na literatura não demonstraram associações entre a CTA e as performances anaeróbias de nadadores $(4,33,43,45)$, evidenciando a necessidade de pesquisas empenhadas em investigar o significado da CTA como preditor de performance em natação. Desse modo, o objetivo do presente estudo foi comparar a VC com o limiar anaeróbio e verificar as relações entre as variáveis aeróbias (LAN e VC) e anaeróbia (CTA) com o desempenho de nadadores nas distâncias de $15 \mathrm{~m}$, $25 \mathrm{~m}, 50 \mathrm{~m}, 100 \mathrm{~m}, 200 \mathrm{~m}$ e $400 \mathrm{~m}$ nado crawl.

\section{METODOLOGIA}

\section{Participantes}

Foram avaliados 8 nadadores de nível nacional com idade de $17 \pm 1$ anos, massa corporal de $64,46 \pm 4 \mathrm{~kg}$, estatura de $173 \pm 3 \mathrm{~cm}$ e tempo mínimo de natação competitiva de 5 anos. Os participantes somente foram confirmados após manifestação em termo de consentimento, aprovado pelo comitê de ética da Unesp, campus de Rio Claro, assinado pelos pais e técnicos das equipes.

\section{Testes}

Os testes tiveram duração total de 3 dias. No dia 1 foi determinado o Limiar anaeróbio (LAN), enquanto que nos dias 2 e 3 foram obtidas as performances 
máximas (Pmáx). Previamente ao início dos testes, os nadadores realizaram um período de aquecimento típico ( $1000 \mathrm{~m})$ em ritmo moderado, sendo composto de esforços utilizando os 4 estilos (borboleta, costas, peito e $\mathrm{crawl}$ ) com predominância para o nado crawl.

\section{Determinação do limiar anaeróbio (LAN)}

Para determinação do Lan foi utilizado o protocolo validado por Pereira et al (35). Nesse protocolo os atletas realizaram três nados de $400 \mathrm{~m}$ com intensidades respectivas de 85,90 , e $100 \%$ da melhor performance do atleta para o percurso, com 3 minutos de pausa entre os nados. Ao final dos exercícios foram coletadas amostras de sangue $(25 \mu \mathrm{l})$ do lóbulo da orelha em tubos capilares calibrados, sendo transferidas para tubos Eppendorf de 1,5 ml, contendo $50 \mu \mathrm{l}$ de NaF (fluoreto de sódio - 1\%). O homogenado foi injetado $(25 \mu \mathrm{l})$ em Lactímetro Eletro Químico YSI (1500 SPORT, YSI, Ohio, EUA) para a determinação da concentração de lactato. As coletas foram realizadas 1 minuto após o término de cada um dos dois primeiros nados e 1,3 e 5 minutos após o final do terceiro. No terceiro nado, considerou-se a concentração de lactato mais elevada dentre as três amostras (1, 3 e 5 minutos). Para cada nado, foi determinada a velocidade média e sua respectiva concentração de lactato. Por ajuste de curva de crescimento exponencial, utilizando programa Origin 6.0, determinou-se a velocidade correspondente a 3,5 $\mathrm{mM}$ de lactato (velocidade de limiar anaeróbio).

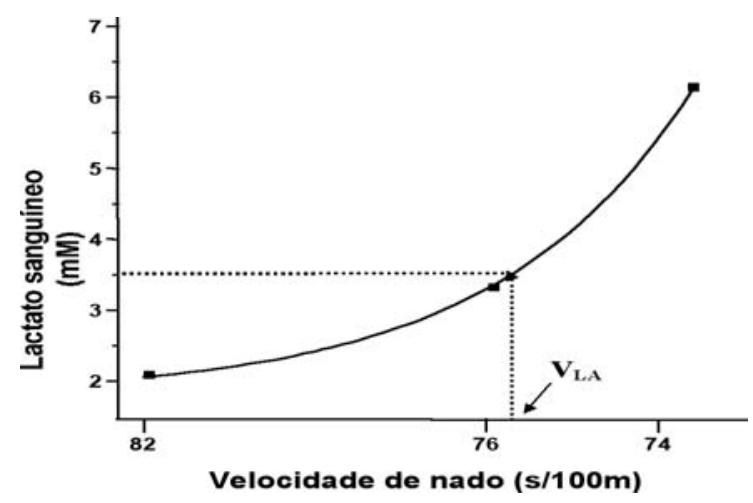

Figura 1. Determinação da velocidade correspondente ao limiar anaeróbio (VLAN) de um atleta durante teste incremental de natação.
Determinação da velocidade crítica (VC), capacidade de nado anaeróbio (CTA) e performances máximas Para determinação das performances máximas foram realizados cinco esforços máximos randomicamente estabelecidos nas distâncias de $15 \mathrm{~m}, 25 \mathrm{~m}, 50 \mathrm{~m}$, $100 \mathrm{~m}$ e $400 \mathrm{~m}$ em estilo crawl durante 2 dias e separados por períodos mínimos de repouso de 2 horas. A VC e a CTA foram determinadas utilizando as velocidades obtidas nas distâncias de $200 \mathrm{~m}$ e $400 \mathrm{~m}$, proposto por Wakayoshi et al. (49) e modificado por Kokubun (19). Os valores de distância e tempo foram submetidos ao procedimento de regressão linear para estimativa da VC e CTA (modelo distância-tempo). O coeficiente angular de cada uma das regressões individuais e o coeficiente linear dos sujeitos representaram a velocidade crítica $(\mathrm{VC})$ e a capacidade de nado anaeróbio (CTA), respectivamente (Figura 2).

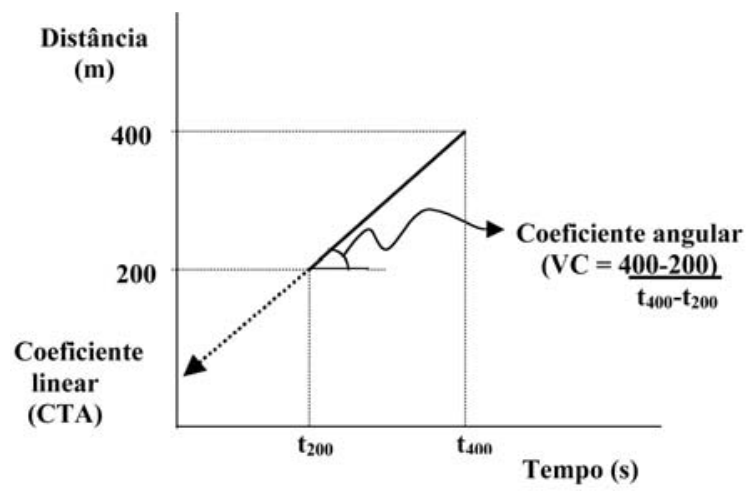

Figura 2. Regressão linear para determinação da velocidade crítica (VC) e capacidade de nado anaeróbio (CTA).

\section{Tratamento estatístico}

As relações entre os testes invasivo, não invasivo e de performances, foram obtidas a partir de análise de correlação de Pearson. Os valores entre limiar anaeróbio e velocidade crítica foram comparados pelo teste " $\mathrm{t}$ " de student para amostras dependentes. Em todos os casos, o nível de significância foi préfixado para $\mathrm{p}<0,05$.

\section{RESULTADOS}

Os valores de limiar anaeróbio (LAN), velocidade crítica (VC), capacidade de nado anaeróbio (CTA) e 
das performances máximas em nado crawl estão apresentados nas tabelas 1 e 2 . Não foi observada diferença significativa entre a VC e o LAN (Tabela 1). Além disso, essas variáveis foram altamente correlacionadas (Figura 3).

Tabela 1. Valores médios \pm desvio padrão do limiar anaeróbio ( $L A N$ ), velocidade crítica (VC) e capacidade de trabalho anaeróbio (CTA).

\begin{tabular}{lll}
\hline LAN $(\mathrm{m} / \mathrm{s})$ & VC $(\mathrm{m} / \mathrm{s})$ & CTA (m) \\
$1,37 \pm 0,5$ & $1,38 \pm 0,7$ & $23,7 \pm 10,5$ \\
\hline
\end{tabular}

Tabela 2. Valores médios \pm desvio padrão das performances máximas ( $\mathrm{m} / \mathrm{s}$ ] nas distâncias de $15 \mathrm{~m}, 25 \mathrm{~m}, 50 \mathrm{~m}, 100 \mathrm{~m}, 200 \mathrm{~m}$ e $400 \mathrm{~m}$ nado crawl.

\begin{tabular}{llllll}
\hline P15 & P25 & P50 & P100 & P200 & P400 \\
$2,09 \pm$ & $1,97 \pm$ & $1,94 \pm$ & $1,73 \pm$ & $1,57 \pm$ & $1,48 \pm$ \\
0,09 & 0,07 & 0,05 & 0,07 & 0,05 & 0,05 \\
\hline
\end{tabular}

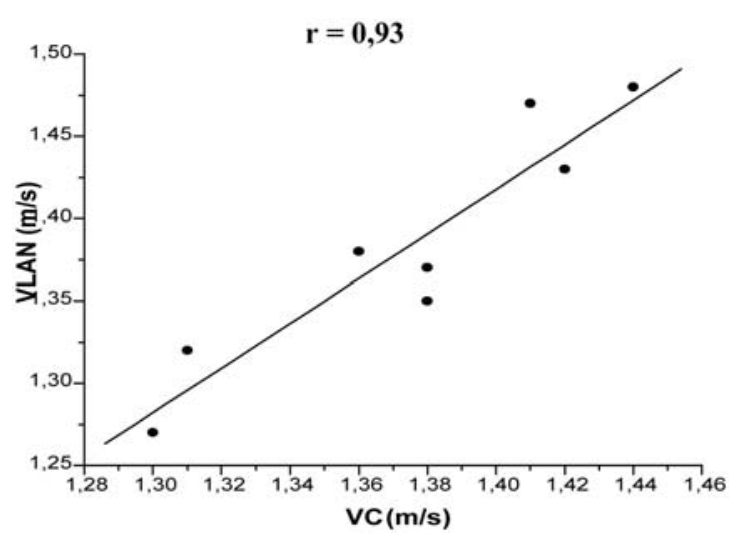

Figura 3. Correlação entre velocidade correspondente ao limiar anaeróbio (VLAN) e velocidade crítica (VC).

O LAN e a VC correlacionaram-se altamente com a performance de $400 \mathrm{~m}$. No entanto, a CTA não apresentou correlações significativas com nenhuma das performances de nado (Tabela 3).

Tabela 3. Correlações entre o limiar anaeróbio (LAN), velocidade crítica (VC) e capacidade de nado anaeróbio (CTA) com as performances máximas ( $\mathrm{m} / \mathrm{s}$ ) nas distâncias de $15 \mathrm{~m}, 25 \mathrm{~m}, 50 \mathrm{~m}, 100 \mathrm{~m}, 200 \mathrm{~m}$ e $400 \mathrm{~m}$ nado crawl.

\begin{tabular}{lllllll}
\hline & P15 & P25 & P50 & P100 & P200 & P400 \\
LAN & 0,43 & 0,68 & 0,61 & 0,55 & 0,50 & $0,96^{*}$ \\
{$[\mathrm{~m} / \mathrm{s}]$} & & & & & & \\
VC $(\mathrm{m} / \mathrm{s})$ & 0,19 & 0,59 & 0,63 & 0,46 & 0,20 & $0,91^{*}$ \\
CTA $(\mathrm{m})$ & 0,32 & 0,09 & 0,05 & 0,06 & 0,36 & $-0,55$ \\
\hline
\end{tabular}

* Indica correlação significativa para $p<0,05$.

\section{DISCUSSÃO}

A diferença não significativa $(\mathrm{p}<0,05)$, juntamente com as elevadas correlações observadas entre o LAN e VC $(r=0,93)$, reforça os achados de Dekerle et al. (4), Kokubun (19), Papoti et al. (33), Toussaint et al. (45), Vilas Boas (47) e Wakayoshi et al. $(48,49)$, ao sugerirem a utilização da VC como um índice para determinar a capacidade aeróbia de nadadores. Essa afirmação é ainda fortalecida pelas elevadas correlações $(p<0,01)$ entre VC e a performance de $400 \mathrm{~m}$, esforço em que se observa significativa participação do componente aeróbio.

Para identificação do LAN no presente estudo utilizaram-se pausas de apenas 3 minutos entre os esforços, visando a otimização do tempo nos testes. Para isso adotou-se a concentração fixa de $3,5 \mathrm{mM}$ de lactato (35) e não a de $4,0 \mathrm{mM}$, como geralmente é utilizado $(11,39)$. A utilização dessa concentração contraria Heck et al. (11), que sugerem a concentração fixa de 3,5 mM apenas para protocolos com estágios com duração de até 3 min, inferiores aos utilizados nesse estudo (4 a $5 \mathrm{~min}$ ). O uso da concentração fixa de $4 \mathrm{mM}$, sugerida por esses autores quando a duração dos estágios for de 5 minutos, parece superestimar o LAN na natação, se a pausa entre os esforços incrementais for pequena, provavelmente devido à existência de efeitos residuais do metabolismo e da fadiga específica dos estágios anteriores, visto que os testes incrementais para determinação do LAN podem ser considerados protocolos dependentes (18).

Estudos experimentais e de revisão da literatura demonstraram associações significativas entre a CTA e o teste de Wingate (9), o trabalho intermitente total acumulado (15) e a produção de ATP muscular (8), além de demonstrarem contribuição significativa da CTA no desempenho em corridas acima de $8 \mathrm{Km}$ (2). Também foi demonstrada a sensibilidade da CTA aos efeitos do treinamento intenso (14), resistido (1) e a suplementação de creatina (41), evidenciando a possibilidade desse parâmetro ser utilizado como medida indireta na avaliação e predição de performances anaeróbias.

Interessante notar que os valores de CTA no presente estudo, ao contrário da maioria das investigações citadas anteriormente, não apresentaram correlações significativas com nenhuma das performances máximas. 
Guglielmo e Denadai (10) não encontraram correlações entre a CTA de nadadores com a potência média determinada durante esforços máximos de 30 segundos em ergômetro de braço isocinético. Papoti et al. (33), utilizando um sistema de nado atado, verificaram correlação significativa entre a força média (FNA) durante esforços máximos de 30 segundos e performances de $100 \mathrm{~m}$ e $200 \mathrm{~m}$ nado crawl, mas não entre FNA e CTA obtida através do intercepto-y da relação linear distância versus tempo.

Soares et al. (43), após investigarem a aplicação da CTA (determinada através da relação obtida entre a distância pré-fixada pelo tempo de nado), como indicador da capacidade anaeróbia e sua sensibilidade ao longo do processo de treinamento em nadadores infantis e adultos, concluíram que a CTA não fornece informações consistentes sobre a capacidade anaeróbia de nadadores, independentemente da faixa etária considerada.

Dekerle et al. (4) também não constataram correlação significativa entre a CTA e a máxima distância anaeróbia em nadadores e sugeriram a não utilização deste parâmetro para controlar variáveis anaeróbias. Uma possível explicação para a contradição observada na literatura, em relação à utilização da CTA como parâmetro preditor de performances anaeróbias, pode ser porque as pesquisas que constataram correlações entre CTA e variáveis anaeróbias foram realizadas em cicloergômetros $(8,14,15)$ e com sujeitos destreinados $(1,14,15)$. Nessas metodologias, a relação utilizada para o procedimento de regressão linear considera o tempo limite (Tlim), enquanto que no modelo de distância fixa proposto por Wakayoshi et al. (49) implica que o nadador, teoricamente, não seja capaz de sustentar a velocidade de nado implementada durante os esforços, em qualquer distância superior à pré-fixada $(200 \mathrm{~m}$ e 400m). Essa hipótese parece limitar a utilização do modelo, principalmente sob o aspecto anaeróbio, pois alguns nadadores são capazes de sustentar a velocidade de nado obtida nas distâncias de $200 \mathrm{~m}$ e $400 \mathrm{~m}$ por mais alguns metros, provavelmente devido à capacidade de tolerância à acidose.

Entretanto, Toussaint et al. (45) investigaram se os conceitos de potência crítica e de CTA poderiam ser utilizados para avaliar as capacidades aeróbia e anae- róbia de nadadores. Para isso, os autores baseados em estudos prévios e em avaliações realizadas no swimming flume, desenvolveram um modelo matemático relacionando a mecânica e a energética envolvida no nado crawl. Também modelaram a liberação de energia aeróbia e anaeróbia em relação ao tempo de nado. Os autores concluíram que, embora a velocidade crítica seja um indicativo do sistema aeróbio, a CTA sofre influência por ambas variações da energia proveniente dos sistemas anaeróbio e aeróbio, não fornecendo uma estimativa real da capacidade anaeróbia. Além disso, sua reprodutibilidade é baixa $(\mathrm{r}=$ 0,62 ) quando comparada aos valores de potência crítica $(0,92)(7)$.

Outra hipótese para explicar a não representatividade da CTA como parâmetro preditor de performance de nadadores é que a maioria das pesquisas que investigaram a CTA de nadadores utilizou, assim como no presente estudo, o modelo de 2 velocidades proposto por Wakayoshi et al. (49). Como nesse protocolo os parâmetros de VC e CTA são obtidos somente pela realização de dois esforços máximos (200 m e $400 \mathrm{~m}$ ), pequenas variações na velocidade de nado podem resultar em significativas alterações no intercepto-y.

Hill et al. (12) relataram que a CTA é um parâmetro sensível para mensurar a capacidade anaeróbia somente quando esta apresentar um erro padrão do coeficiente linear inferior a $10 \%$. Se a CTA for obtida com a realização de vários esforços, teoricamente será possível determinar o erro do coeficiente linear (intercepto-y) e conseqüentemente obter maior precisão nos valores de CTA. No entanto, Bishop e Jenkins (1) encontraram elevada correlação negativa $(r=-0,94)$ entre alterações na potência crítica (PC) e a CTA após 6 semanas de treinamento resistido, evidenciando uma limitação ao modelo linear de determinação da PC.

\section{CONCLUSÃO}

Pode-se concluir que a VC é um parâmetro confiável na avaliação da capacidade aeróbia e na predição da performance de $400 \mathrm{~m}$ de nadadores. No entanto, a CTA obtida pelo intercepto-y da linearização, distância pré-fixada e tempo de nado não representa um parâmetro válido na predição de performance nas distâncias entre $15 \mathrm{~m}$ e $400 \mathrm{~m}$ em natação. 


\section{Agradecimentos}

Os autores agradecem o apoio da Fundação de Amparo à Pesquisa do Estado de São Paulo FAPESP (Processo n ${ }^{\circ}$ 01/08295-2) e do CNPq (Processo no130841/2003-0) na realização deste trabalho.

\section{CORRESPONDÊNCIA}

Marcelo Papoti

Laboratório de Biodinâmica, UNESP Rio Claro, SP Av. 24 A 15-15, Bela Vista, Rio Claro, SP 13506-900 Brasil papoti@ig.com.br

\section{REFERÊNCIAS}

1. Bishop D, Jenkins DG. (1996). The influence of Resistance Training on the Critical Power Function \& Time to Fatigue at Critical Power. The Australian Journal of Science and Medicine in Sport 28: 101-105.

2. Bulbulian R, Wilcox AR, Darabos BI. (1986). Anaerobic contribution to distance running performance of trained cross-country athletes. Medicine and Science in Sports and Exercise 18: 107 - 113.

3. Costill DI, Reifield F, Kirwan J, Thomas R. (1986). A computer based system for the measurement of force and power furing front crawl swimming. Journal of Swimming Research 2: 16 - 19.

4. Dekerle J, Sidney M, Hespel, MJ, Pelayo P. (2002). Validity and Reliability of Critical Speed, Critical Stroke Rate, and Anaerobic Capacity in relation to Front Crawl Swimming Performances. International Journal of Sports Medicine 23: 9398.

5. Deminice R, Prado JRMV, Papoti M, Zagatto A. (2003). Utilização de métodos não-invasivos como indicador da capacidade aeróbia e da performance em natação competitiva. Revista Brasileira de Ciência e Movimento (Suplemento), 130.

6. Fry RW, Morton AR, Garcia-Webb P, Crawford GPM, Keast D. (1994). Psychological and immunological correlates of acute overtraining. British Journal of Sports Medicine 28: 241 -246 .

7. Gaesser GA e Wilson IA. (1988). Effects of continuous and interval training on the parameters of the powerendurance time relationship for high-intensity exercise. International Journal of Sports Medicine 9: 417 - 21.

8. Green S, Dawson BT, Goodman, C, Carey MF. (1994). Yintercept of the maximal work-duration relationship and anaerobic capacity in cyclists. European Journal of Applied Physiology 69: 550 - 556.

9. Green, S. (1995). Measurement of Anaerobic Work Capacities in Humans. Sports Medicine 19: 32-42.

10. Guglielmo IGA, Denadai BS. (1999). Correlação do teste de Wingate de braço com a capacidade de trabalho anaeróbio determinada através do conceito de velocidade crítica na natação. Motriz (Suplemento) 5: 92.

11. Heck H, Mader A, Hess G, Mucke S, Muller R, Hollmann W. (1985). Justification of the $4 \mathrm{mmol} / 1$ lactate threshold. International Journal Sports Medicine 6:117 - 30.

12. Hill DW, Jimmy C, Smith C. (1994). A method to ensure the accuracy of estimates of anaerobic capacity derived using the critical power concept. The Journal of Spots Medicine and Physical Fitness 34:23-37.

13. Hooper SL, Mackinnon IT, Ginn EM. (1998). Effects of three tapering techniques on the performances, forces and psychometric measures of competitive swimmers. European Journal of Applied Physiology 78: 258 - 263.

14. Jenkins DG, Quigley BM. (1993). The influence of highintensity exercise on the Wlim-Tlim relationship. Medicine and Science in Sports and Exercise 25: 275 - 282.

15. Jenkins DG, Quigley BM. (1991). The y-intercept of the critical power function as a measure of anaerobic work capacity. Ergonomics 31: 1413 -1419.

16. Jeukendrup AE, Hesselink MK. (1994). Overtraining what do lactate curves tell us? British Journal of Sports Medicine 28: 239 - 240. 
17. Johns, RA, Houmard AJ, Kobe WR, Hortobágyi T, Bruno JN, Wells MJ, Shinebarger HM. (1992). Effects of taper on swim power, stroke distance, and performance. Medicine and Science in Sports and Exercise 24: 1141 -1146.

18. Kiss MAPDM, Fleishmann E, Cordani IK, Kalinovsky F, Costa R, Oliveira FR, Gagliardi JFI. (1995). Validade da velocidade de limiar de lactato de $3,5 \mathrm{mmol} / \mathrm{L}^{-1}$ identificada através de teste em pista de atletismo. Revista Paulista de Educação Fisica 9:16-25.

19. Kokubun E. (1996). Velocidade crítica como estimador do limiar anaeróbio na natação. Revista Paulista de Educação Física 10: 5 - 20.

20. Lehmann M, Baumgart P, Wiesenack C. (1992). Trainingovertraining: influence of a defined increase in training volume $v s$ training intensity on performance, catecholamines and some metabolic parameters in experienced middle and long-distance runners. European Journal of Applied Physiology 64: 169 - 177.

21. Mader A, Heck HA. (1986). Theory of the metabolic origin of anaerobic threshold. International Journal of Sports Medicine 7: 45 - 65.

22. Madsen O, Lohberg M. (1987). The lowdon on lactates. Swimming Technique 24: 21 - 26.

23. Maglischo EW, Maglischo EW, Sharp RI, Zier DJ, Katz A. (1984). Tethered and nontethered crawl swimming. Sports Biomechanics 163 - 176.

24. Maglischo EW. (1999). Nadando ainda mais rápido. São Paulo-SP: Ed. Manole.

25. Marinho PC, Andries O Jr. (2001). Avaliação da força propulsora do nadador: validação e reprodutibilidade de uma metodologia específica. Revista Brasileira de Ciência e Movimento (Suplemento) 79.

26. Monod H, Scherrer J. (1965). The work capacity of a synergic muscular group. Ergonomics 8: 329 - 337.

27. Moritani T, Nagata A, DeVries HA, Muro M. (1981). Critical power as a measure of physical work capacity and anaerobic threshold. Ergonomic 24:339-350.

28. Mujika I, Chatard JC, Busso T, Geyssant A, Barale F, Lacoste L. (1995). Effects of Training on Performance in Competitive Swimming. Canadian Journal of Applied Physiology 20: 395 - 406.

29. Neufrer PD, Costill D, Fielding RA, Flynn MG, Kirwan JP. (1987). Effects of reduced training on muscular strength and endurance in competitive swimmers. Medicine and Science in Sports and Exercise 19: 486 - 490.

30. Olbrecht J, Madsen O, Mader A, Liesen H, Hollmann W. (1985). Relationship between swimming velocity and lactic concentration during continuous and intermittent training exercises. International Journal of Sports Medicine 2: 74 77.

31. Overend TJ, Cunningham DA, Paterson DH, Smith WDF. (1992). Physiological responses of young and eldrey men to prolonged exercise at critical power. European Journal of Applied Physiology 64:187-93.

32. Papoti M, Martins LEB, Cunha SA, Freitas Jr PB, Gobatto C. (2002). Effects of taper on swimming force and performance after a 10-wk training program. In Proceedings of the $7^{\text {th }}$ Annual Congress of the European College of Sport Science, 470.

33. Papoti M, Martins LEB, Cunha AS, Zagatto AM, Pereira RR, Gobatto CA. (2003). Validade na determinação das capacidades aeróbia e anaeróbia de nadadores. Motriz 9: 56.
34. Pelayo P, Mujika I, Sidney M, Chatard J. (1996). Blood lactate recovery measurements, training, and performance during a 23-week period of competitive swimming. European Journal of Applied Physiology 74: 107 - 113.

35. Pereira RR, Zagatto AM, Papoti M, Gobatto CA. (2002). Validação de dois protocolos para determinação do limiar anaeróbio em natação. Motriz 8: 63 - 68.

36. Pyne BD, Lee HE, Swanwick, KM. (2001). Monitoring the lactate threshold in world-ranked swimmers. Medicine and Science in Sports and Exercise 33: 291 - 297.

37. Raglin JS, Koceja DM, Stanger JM, Harms CA. (1996). Mood, neuromuscular function, and performance during training in female swimmers. Medicine and Science in Sports and Exercise 28: 372 - 377.

38. Sharp RL, Troup JP, Costill DL. (1982). Relationship between power and sprint freestyle swimming. Medicine and Science in Sports and Exercise 14: 53 - 56.

39. Sjodin B, Jacobs I. (1981). Onset of blood accumulation and marathon running performance. International Journal Sports Medicine 2:23-6.

40. Smith DJ, Norris RS, Hogg MJ. (2002). Performance Evaluation of Swimmers: Scientific Tools. Sports Medicine 32: 539 - 554.

41. Smith JC. (1998). Effect of oral creatine ingestion on parameters of the work rate-time relationship an time to exhaustion in high-intensity cycling. European Journal of Applied Physiology 77: 360 - 365.

42. Snyder AC, Jeukendrup AE, Hesselink MKC, Kuipers H, Foster CA. (1993). A physiological/psychological indicator of over-reaching during intensive training. International Journal of Sports Medicine 14: 29 - 32.

43. Soares S, Vilar S, Bernardo C, Campos A, Fernandes R, Vilas-Boas JP. (2003). Using data from the critical velocity regression line for the estimation of anaerobic capacity in infant and adult swimmers. Revista Portuguesa de Ciências do Desporto 3:73-118.

44. Tokmakidis PS, Léger AL, Pilianidis, C. T. (1998). Failure to obtain a unique threshold on the blood lactate concentration curve during exercise. European Journal Applied Physiology 77: 333 - 342.

45. Toussaint HM, Wakayoshi K, Hollander PA, Ogita F. (1998). Simulated front crawl swimming performance related to critical speed and critical power. Medicine and Science in Sports and Exercise 30: 144 - 151.

46. Trappe S, Costill D, Thomas R. (2001). Effect of swim taper on whole muscle and single muscle fiber contractile properties. Medicine and Science in Sports and Exercise. 32: 48 $-56$.

47. Vilas Boas JP, Lamares JP, Fernandes R, Duarte JA. (1997). Relationship between anaerobic threshold and swimming critical speed determined with competition times. In Abstracts book of the FIMS $9^{\text {th }}$ European Congress of Sports Medicine, Porto, 88-91.

48. Wakayoshi K, Ikuta K, Yoshida T, Udo M, Moritani T, Mutoh Y, Miyashita M. (1992). Determination and validity of critical velocity as an index of swimming performance in the competitive swimmer. European Journal of Applied Physiology 64: 153 - 157.

49. Wakayoshi K, Yoshida T, Udo M, Harada T, Moritani T, Mutoh Y, Miyashita, M. (1993). Does critical swimming velocity represent exercise intensity at maximal lactate steady state? European Journal of Applied Physiology 66: 90 - 95. 